\title{
Title: Tidal triggering of earthquakes suggests poroelastic behavior on the San Andreas Fault
}

Authors: Andrew A. Delorey ${ }^{1 *}$, Nicholas J. van der Elst ${ }^{2}$, Paul A. Johnson ${ }^{1}$ 


\section{Abstract}

Tidal triggering of earthquakes is hypothesized to provide quantitative information regarding the fault's stress state, poroelastic properties, and may be significant for our understanding of seismic hazard. To date, studies of regional or global earthquake catalogs have had only modest successes in identifying tidal triggering. We posit that the smallest events that may provide additional evidence of triggering go unidentified and thus we developed a technique to improve the identification of very small magnitude events. We identify events applying a method known as inter-station seismic coherence where we prioritize detection and discrimination over characterization. Here we show tidal triggering of earthquakes on the San Andreas Fault. We find the complex interaction of semi-diurnal and fortnightly tidal periods exposes both stress threshold and critical state behavior. Our findings reveal earthquake nucleation processes and pore pressure conditions - properties of faults that are difficult to measure, yet extremely important for characterizing earthquake physics and seismic hazards. 


\section{Background}

Earthquake triggering can occur in response to both quasi-static stress changes and dynamic forcing. Aftershocks are a well-known example of triggering by quasistatic and dynamic stress transfer, while triggering of distant earthquakes by seismic waves is an example of strictly dynamic triggering (Delorey et al., 2015; Gomberg et al., 2004; Gonzalez-Huizar et al., 2012; Hill et al., 1993). Earthquake triggering has the potential to be extremely valuable in probing stress state characteristics such as critical state (near failure) conditions (Brinkman et al., 2015; Brodsky and van der Elst, 2014; Johnson et al., 2013; van der Elst et al., 2013). Triggering by Earth tides can be particularly informative since tides are always present and observations can be stacked in time, in contrast to dynamic earthquake triggering which can occur only during or after the passage of large amplitude seismic waves.

Earth tides are caused by the gravitational pull of the sun and moon, which induce periodic stresses related to the rotation of the Earth relative to the sun and moon (semi-diurnal, $\sim 12$ hours and diurnal, 24 hours). A longer-period modulation of these cycles arises due to the orbit of the moon around the Earth (fortnightly, $\sim 14.7$ days). Earth tides impart both normal and shear stresses on fault surfaces in the upper crust. During tidal-induced periods of increasing Coulomb stress on a fault, the stressing rate is much higher than the long-term tectonic stressing rate determined from the average stressing rate between $M_{w} 6$ earthquakes near Parkfield, California (Agnew, 1997; Kim and Dreger, 2008). Tidal triggering of earthquakes has been observed locally preceding large earthquakes (Tanaka, 2010, 2012; Tanaka et al., 2002b), and also in global or regional datasets that span longer periods of time (Cochran et al., 2004; Metivier et al., 
2009; Tanaka et al., 2002a; Tsuruoka et al., 1995). Tides also trigger earthquakes at volcanoes (Emter, 1997; Mcnutt and Beavan, 1981; Rydelek et al., 1988) and ocean tides (Stroup et al., 2007; Tolstoy et al., 2002; Wilcock, 2001, 2009) may trigger earthquakes in ocean basins.

Continental crust is thought to be critically stressed (Townend and Zoback, 2000) meaning that faults near failure are ubiquitous. If this is true, then we might expect earthquake triggering to be widespread in response to transient stresses associated with tides and large amplitude seismic waves; however this is not observed (Vidale et al., 1998). We propose that observations of the tidal triggering of earthquakes are scarce because most earthquakes and therefore most triggered earthquakes are small and below detection limits. The barrier to observing tidally triggered earthquakes may simply be a lack of completeness in the catalog. Thus our intent here is to improve detections of small earthquakes to determine if tidal triggering of earthquakes is occurring in regions where it is currently not detected using existing earthquake catalogs. Our test case is a segment of the SAF near Parkfield, California (Fig. 1) because it is well instrumented, has a relatively high ambient seismicity rate, and is known to exhibit tidal triggering of low frequency earthquakes (LFEs) (Thomas et al., 2012; van der Elst et al., 2016) and non-volcanic tremors (NVTs) (Thomas et al., 2009) in the middle crust. 


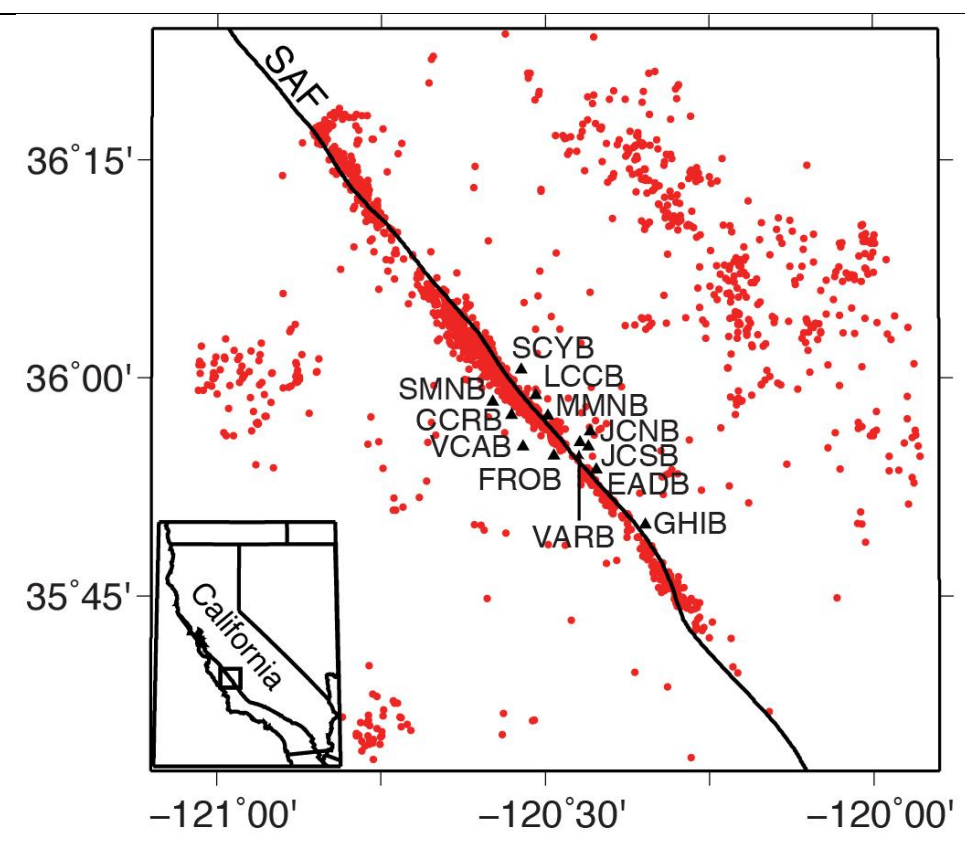

Fig. 1. Map. Red circles represent earthquakes from the NCSN catalog in calendar years 2012-2014 that are within $50 \mathrm{~km}$ of Parkfield, California and $50 \mathrm{~km}$ of the SAF. The black curve labeled "SAF" represents the San Andreas Fault. The labeled black triangles represent the stations of the HRSN used in this study.

\section{Earthquake Catalogs}

Traditional earthquake catalogs like that provided by the Northern California Seismic Network (NCSN) require a sufficient number of phase arrivals in order to estimate location and magnitude, which limits their completeness. Tidal correlation is suggested, but not statistically significant in the NCSN catalog (Fig. S1). We hypothesize that its significance could be limited by the stringency of the catalog location requirements. Thus, we develop a catalog in which we emphasize only detection and discrimination of phase arrivals so that the occurrence of smaller events 
may be cataloged, even when a precise location cannot be obtained (see examples in Figs. 2 and S2). Discrimination in this context means discriminating local, regular earthquakes from distant earthquakes, non-earthquake sources on Earth's surface, and deeper LFEs and NVTs. We search for earthquakes by looking for seismic energy arriving across the NCSN that is consistent with emissions from local earthquakes.

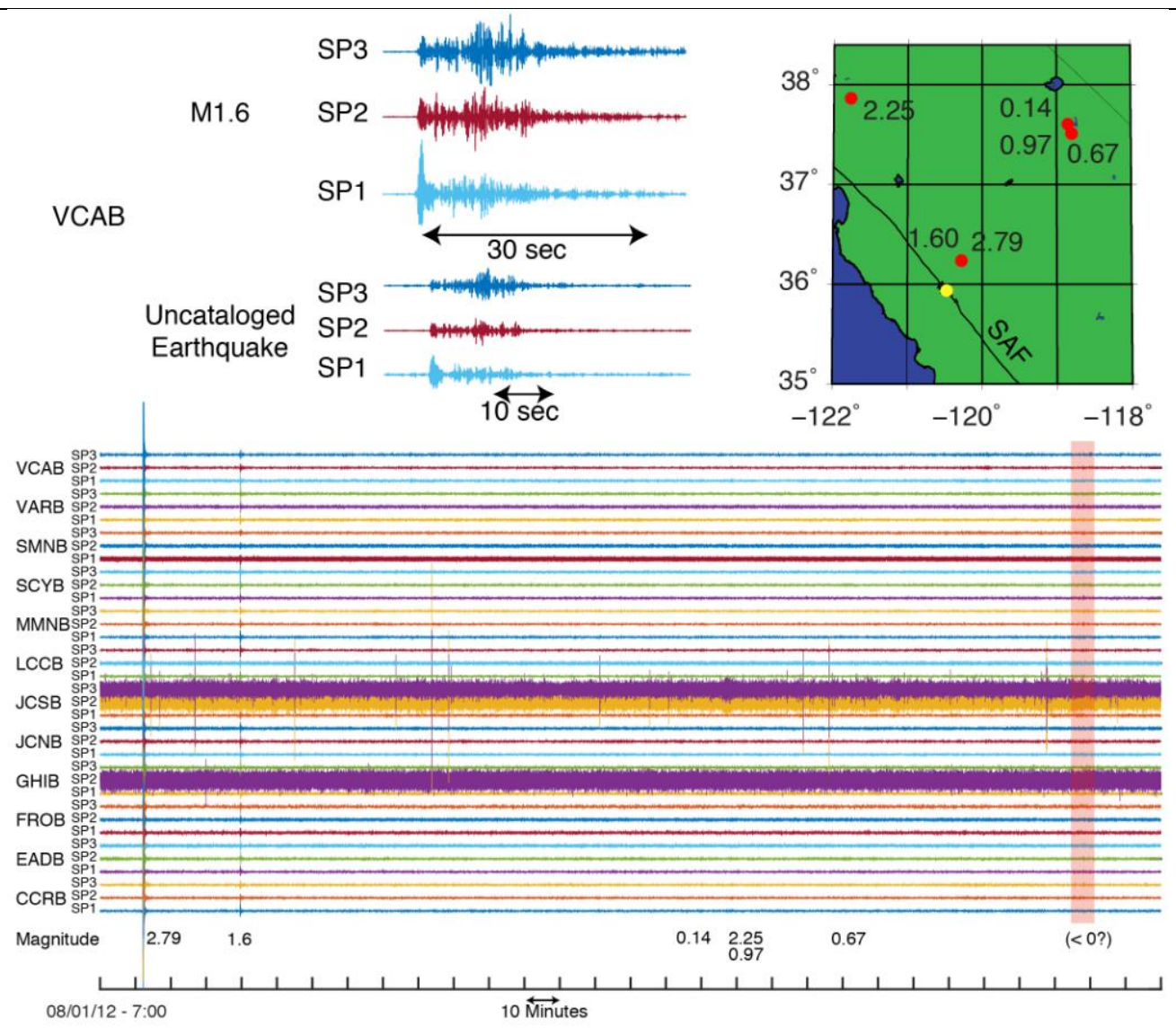

Fig. 2. Example of detected earthquake not present in the NCSN catalog. Shown are six earthquakes with magnitudes of $2.79,1.60,0.14,2.25,0.97$, and 0.67 that are found in the NCSN catalog with an additional uncataloged earthquake found using our method. At upper left is a zoom of the traces from station VCAB with $5 \mathrm{~Hz}$ highpass filter and relative amplitudes preserved. The uncataloged earthquake whose magnitude is likely $<0$ has similar waveforms to the cataloged M1.6 earthquake. At upper right are 
the locations of the six cataloged earthquakes (red circles) shown on the SAF. Some earthquakes are nearly co-located. Using our method, we detect events (red circles) on or near the SAF near Parkfield (yellow circle), but not those farther away (magnitudes $2.25,0.14,0.97$, and 0.67$)$. At bottom are traces from stations and components indicated at left with high pass filter at $1 \mathrm{~Hz}$. The uncataloged event is shaded red, which is buried in the noise at this pass band. The closest cataloged LFE to this uncataloged regular earthquake is 16 minutes prior to it at 11:22:19.

NVTs are detected by identifying similar, emergent, long-duration waveforms across a seismic array cannot be explained by surface sources or noise (Obara, 2002). Correlation techniques are often used to identify and locate NVTs because they do not have impulsive or easily identifiable phase arrivals (Wech and Creager, 2008). Small earthquakes are similar in the sense that it is difficult to pick phase arrivals when the signal to noise ratio is low even when a signal has obvious earthquake characteristics. These earthquakes are missing from traditional catalogs because though they are often easily identified, they are difficult to characterize. Even with minimal characterization, these earthquakes contain valuable information and are worth cataloging.

We apply a similar approach to finding small earthquakes as others have used to find NVTs (Obara, 2002; Wech and Creager, 2008). We use unique array characteristics of local earthquakes to identify them in continuous data. Crosscorrelating the waveform envelope, we look for energy within the $5-10 \mathrm{~Hz}$ band that has apparent velocities consistent with body waves emanating from sources within the local crust. Waves from regional and distant sources are highly attenuated in this band. The highest amplitude waves from non-earthquake, surface sources have apparent 
118 velocities of surface waves and in the $5-10 \mathrm{~Hz}$ band have very small displacements at 119 the depths of the borehole instruments of the NCSN. Also, since we do not use station

120 pairs closer together than $5 \mathrm{~km}$, only very energetic non-earthquake sources will be

121 recorded on two or more instruments (See methods for a full description of the

122 earthquake detection method and the Supplementary data for our complete catalog.)

123 Our coherence-derived earthquake catalog covers the calendar years 2012-2014

124 and contains 6735 earthquakes. In addition to the earthquakes we detect, we add 206

125 earthquakes from the NCSN catalog that are within $50 \mathrm{~km}$ of Parkfield and $5 \mathrm{~km}$ of the

126 SAF that do not correspond to an earthquake in our base catalog, resulting in a total

127 catalog of 6941 earthquakes. During the same period of time there are 1654

128 earthquakes in the NCSN earthquake catalog within $50 \mathrm{~km}$ of Parkfield and $5 \mathrm{~km}$ of the

129 SAF. We do not determine a location for our detections other than to note the closest

130 station. However by comparing our detections to matching detections in the NCSN

131 earthquake catalog, we estimate that most ( $>90 \%)$ are also within about $50 \mathrm{~km}$ of

132 Parkfield. $75 \%$ of the events in the NCSN catalog within $50 \mathrm{~km}$ of Parkfield are within 5

$133 \mathrm{~km}$ of the SAF (Fig. S3), and thus we assume the same for our catalog. Our detections

134 with matching entries in the NCSN earthquake catalog are not biased with regard to the

135 distribution of depths found in the full NCSN catalog for the study period and region,

136 which have a mean depth of $6.4 \mathrm{~km}$ and a median depth of $5.2 \mathrm{~km}$.

137 For each earthquake in our catalog, we assign a phase for both the semi-diurnal 138 cycle and the fortnightly cycle (Fig S4). An important point is that the total tidal stress

139 time series is not a simple sinusoid. When we refer to the semi-diurnal phase, what we 140 mean is the phase associated with the total tidal stress time series, whose period is 
141 dominated by the semi-diurnal period of $\sim 12.4$ hours. In any given cycle, the apparent

142 period and phase is perturbed somewhat by the influence of other tidal components

143

144 whose periods are close to 12 or 24 hours. However, over long periods of time the average period is the semi-diurnal period. We determine the phase from the entire tidal stress time series because faults do not feel each tidal component independently. In the case of the fortnightly cycle, we use the timing of lunar phases to determine the phase.

We here consider temporal processes that could mimic tidally modulated clustering but be only coincidently correlated with tidal stresses. The lunar semi-diurnal component $\left(\mathrm{M}_{2}\right)$ has the largest amplitude with more than twice that of the solar semidiurnal component $\left(S_{2}\right)$. The phase of the $\mathrm{M}_{2}$ component dominates the phase of the total stress signal. With a period of $\sim 12.4$ hours, the phase of the $M_{2}$ component progressively shifts relative to daytime and nighttime hours, so over the three year study period there is no net correlation with any diurnal signal anthropogenic or otherwise (see supplemental material). Potential biases introduced by seasonal processes do not affect the total catalog because the duration of our study is three full calendar years. The other manner in which there may be apparent correlation of seismicity with tides is if aftershocks or other clustered events are correlated with tidal stresses but not caused by tidal stresses. Since our detection catalog contains only the timing of earthquakes, we do not have enough information to apply standard algorithms to remove aftershocks. Instead, we test the possibility that aftershock clustering could invalidate our measurements by creating 10000 synthetic catalogs that contain aftershocks using an ETAS model consistent with California seismicity (Field et al., 
2014) but no explicit periodic forcing. We performed a maximum likelihood estimation (MLE) testing the periodicity of the synthetic catalogs against the periodicity of our catalog. Only 24 of the 10000 synthetic catalogs were more periodic than ours on the semi-diurnal cycle. All of these 24 synthetic catalogs contained a large ( $M>6.8)$ earthquake whose aftershocks dominate the catalog statistics. No catalog with a maximum magnitude less then 6.8 was more periodic than ours. The maximum magnitude earthquake during our study period is 4.5 .

Lastly, we examine how the observed correlation between seismicity and Earth tides varies over time and whether or not some short-duration behavior of the catalog dominates the statistics of the entire catalog. We determined that the observed correlation between Earth tides and seismicity is prevalent throughout the study period. Details of this analysis are in the supplemental materials.

\section{Earth Tides}

Tidally induced strains can be modeled (Agnew, 1997) and with appropriate elastic moduli we can calculate stress at seismogenic depths allowing us to quantify the relationship between stress forcing (changes in Coulomb stress) and earthquake clustering. In the most general case, Skempton's coefficient $(B)$ is a tensor, which measures how much of the normal stress is supported by pore pressures versus the rock matrix. However, we can consider more specific cases for an undrained fault system. Change in Coulomb stress (Beeler et al., 2000) for an anisotropic system and isotropic system, is respectively,

$$
\begin{aligned}
& \Delta C=\Delta \tau+\mu(1-B) \Delta \sigma \\
& \Delta C=\Delta \tau+\mu\left(\Delta \sigma-B \Delta \sigma_{m}\right) .
\end{aligned}
$$


187 where $\Delta C$ is the change in Coulomb stress, $\Delta_{r}$ is change in shear stress, $\mu$ is the

188 coefficient of friction, $\Delta \sigma$ is the change in stress normal to the fault and $\Delta \sigma_{m}$ is the mean

189 change in compressive stress. Positive $\sigma$ is extensional. (A full description of tidal

190 stress calculations can be found in the methods.).

191 4. Results

192 In order to characterize the observed clustering behavior in our catalog we first

193 divide the lunar cycle into halves corresponding to rising and falling fortnightly tides. For

194 events in these two halves we assign a phase value to each earthquake corresponding

195 to its position in the semi-diurnal cycle. Our null hypothesis is that earthquake times

196 correspond to a homogeneous Poisson distribution. We test the hypothesis that

197 earthquakes times are periodic with the same periods as the semi-diurnal and fortnightly

198 cycles and model the periodicity as a nonhomogeneous Poisson process. We then

199 perform a MLE for the parameters of a periodic model for seismicity during rising and

200 falling fortnightly tides with the same period as the semi-diurnal cycle. Finally we

201 perform a likelihood ratio test comparing the periodic (nonhomogeneous) model to the

202 homogeneous model.

203 Strictly speaking our catalog event times do not have a Poisson distribution

204 because in a Poisson distribution there is no dependence of one event on another and

205 our catalog contains aftershocks, which are dependent events. This has an impact on

206 the likelihood values and ratios we calculate. However, we demonstrated with synthetic

207 catalogs that aftershock clustering does not coincidentally produce periodic behavior.

208 So, higher likelihoods calculated for the nonhomogeneous, periodic models are entirely

209 due to better fitting of the tidally driven periodicity. 
For seismicity during rising fortnightly tides we cannot reject the null hypothesis

211 that earthquake times correspond to a homogeneous Poisson distribution (Fig. 3a,

212 S5a). There is no apparent correlation between seismicity and semi-diurnal stress

213 during rising fortnightly tides. Such a correlation either does not exist or is beyond our

214 ability to detect it. However, for seismicity during falling fortnightly tides we reject the

215 null hypothesis with a confidence of 0.986 (Fig. 3b, S5b). We also test the periodicity of

216 all earthquakes with respect to the fortnightly cycle in the same manner, ignoring the

217 semi-diurnal phase. We reject the null hypothesis with a confidence of 0.999 (Fig. 3e,

218 S5c). We find that during falling fortnightly tides, seismicity peaks around the highest

219 Coulomb stresses (peak rate lags peak stress by 5 degrees) within the semi-diurnal

220 tidal cycle. Over the fortnightly cycle, earthquakes are more likely to be found at the

221 beginning of the cycle when absolute peak stress (APS, the highest stress attained up

222 to a point in time) is exceeded most often. A full description of our statistical analysis is 223 in the methods. 


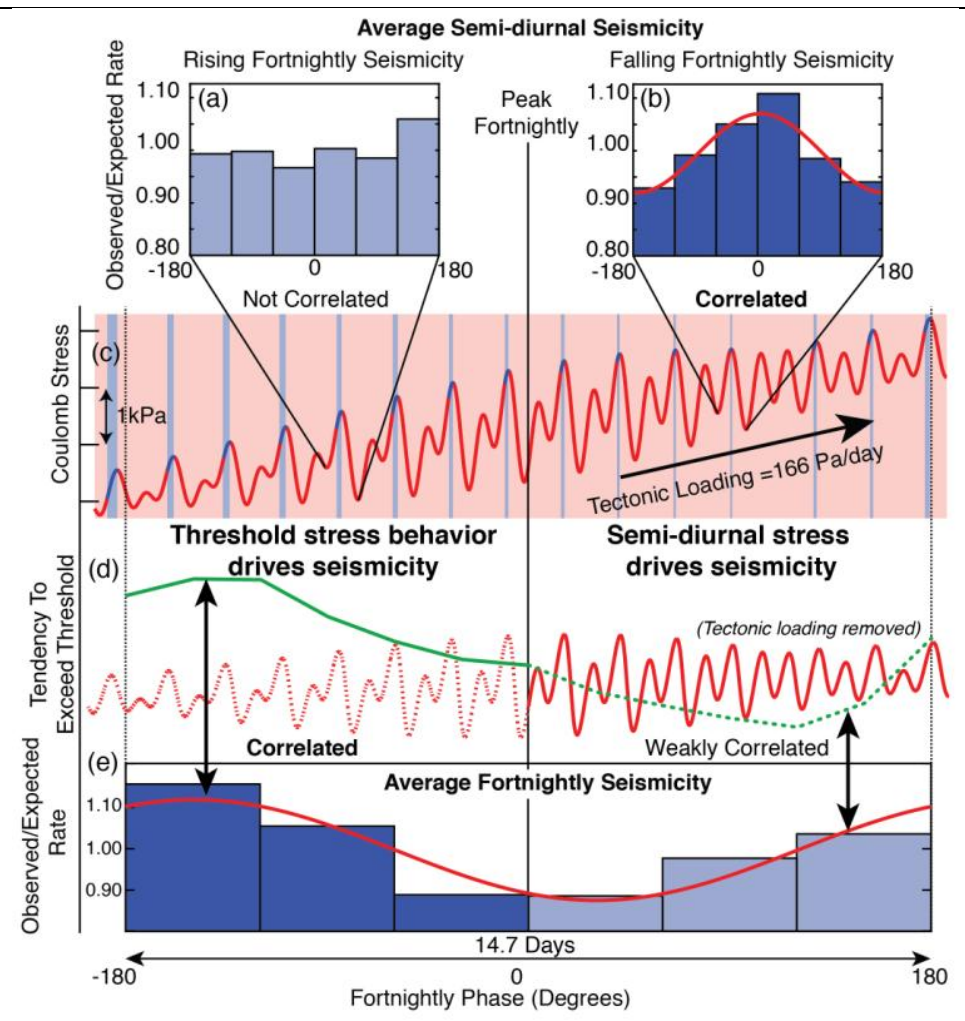

Fig. 3. Observations. Average seismicity as a function of semi-diurnal phase is shown during rising (a) and falling (b) fortnightly tides. Black sub-vertical lines connected to (c) indicate one semi-diurnal cycle (12.4 hours). During rising fortnightly tides (a) seismicity is not correlated and during falling fortnightly tides (b) seismicity is correlated with the semi-diurnal stress cycle whose maximums are at phase zero. The red curve is the maximum likelihood periodic function for seismicity as a function of semi-diurnal phase. (c) Tidally driven Coulomb stress (effective $\mu=0.5$ ) plus tectonic loading on SAF. Light blue regions indicate periods where stress threshold is exceeded while pink regions indicate periods of stress shadowing. (d) Green curve is tendency to exceed stress threshold as a function of fortnightly phase. Red curve is tidally driven Coulomb stress on SAF without tectonic loading. During rising fortnightly tides threshold behavior drives seismicity (green solid curve); during falling fortnightly tides semi-diurnal stress drives seismicity (green dashed curve). (e) Average fortnightly seismicity for falling (blue) and falling (light blue) phase. Red curve is the maximum likelihood periodic function for seismicity as a function of fortnightly phase. 
We use an effective coefficient of friction of 0.5 to produce Fig. 3 because it produces tidally driven Coulomb stress that is dominated by the normal component, although our results change little for effective coefficients of friction between 0.4 and 1 . If the effective coefficient of friction is close to zero then the phase of Coulomb stress is essentially the same as the phase of tidal shear stress and peak seismicity is anticorrelated with peak Coulomb stress. For higher effective coefficients of friction, the phase of Coulomb stress is the same as the phase of tidal normal stress and peak seismicity is positively correlated with peak Coulomb stress. constraints on frictional conditions on the SAF, namely that the contribution of normal stress to the change in Coulomb stress is sufficiently larger than the contribution of shear stress for anisotropic or isotropic conditions, respectively, maximum tidal normal stress $\Delta \sigma \sim 2600 \mathrm{~Pa}, \Delta \sigma_{m} \sim 600 \mathrm{~Pa}$. If additionally the effective coefficient of friction is at least 0.4 then for anisotropic and isotropic conditions,

242 respectively, rising tides, overall seismicity rates are high and earthquakes occur uniformly with

To reiterate, we observe two apparently interdependent behaviors: 1) During respect to the semi-diurnal cycle; 2) During falling fortnightly tides, seismicity rates are 
248 lower, and earthquakes preferentially coincide with the peak semi-diurnal stress. To 249 interpret the relationship between solid earth tides and seismicity we require a model

250 that explains both behaviors. We look to observations and laboratory experiments for

251 guidance in understanding the physical processes in the Earth.

252

253

\section{Discussion and Conclusions}

Experiments and models generally define only narrow conditions in which tidal triggering of earthquakes should be detectable (Bartlow et al., 2012; Beeler and Lockner, 2003; Dieterich, 1987). Two important characteristics of tidal stresses at Parkfield have not been sufficiently explored: (1) tidal stresses are not simple sinusoids and (2) there is likely considerable stress shadowing — periods of time when stresses are below APS. The amount of stress shadowing depends heavily on the background or "tectonic" stressing rate, which can be estimated by the stress drops, afterslip, and inter-event times of large earthquakes (Murray and Langbein, 2006). To determine the average background stressing rate on the SAF near Parkfield we use the average stress drop of the 2004 Parkfield earthquake (Kim and Dreger, 2008) and divide it by the time interval since the previous large earthquake in 1966 to obtain a value of 166 $\mathrm{Pa} /$ day. When combining tectonic and tidal stress two different kinds of stress shadows are produced, the shadow cast by peak semi-diurnal stress over subsequent semidiurnal cycles, and the shadow cast by the peak of the fortnightly cycle on subsequent semi-diurnal cycles (Fig. 3c, 3d). The stress shadowing is so significant with reasonable background stressing rates that APS is only exceeded $\sim 8 \%$ of the time and those times are not evenly distributed over the semi-diurnal or fortnightly cycles. 
If earthquakes only occur when APS is exceeded then they would only occur

during the periods of blue in Fig. $3 c$ and that is not what is observed. Additionally, periods of peak stressing rate are always in a stress shadow so it seems unlikely that peak seismicity should correlate with those times even though that is what is predicted for a purely threshold process (Beeler and Lockner, 2003). Stress shadows have been discussed but not fully explored for their affect on seismicity (e.g. Bartlow et al.(2012) and Thomas et al. (2012). For instance Bartlow et al. (2012) use a simple sinusoid to represent tidal stress and a background-stressing rate such that there are no stress shadows in their experiments. Thus, their results may not be representative of behavior on the SAF at Parkfield.

Rather than propose a new model, we discuss the role of stress shadows and the correlation between stress and seismicity in the context of rate and state friction (Dieterich, 2007) and stress threshold behavior. Existing models make specific predictions regarding stress and poroelastic conditions based on a system's response to periodic stressing (Bartlow et al., 2012; Beeler and Lockner, 2003; Dieterich, 1987; Thomas et al., 2012; Thomas et al., 2009; van der Elst et al., 2016). Even without proposing a new model we can put some constraints on effective normal stress and poroelastic behavior, two important but often-inaccessible properties of a fault.

We consider that the observed difference in semi-diurnal tidal triggering between the rising and falling parts of the fortnightly cycle is related to the presence and distribution of stress shadows (Fig. 3). APS is exceeded most commonly during rising fortnightly tides (Fig. 3d), with long periods of stress shadowing more prevalent during falling fortnightly tides. During some fortnightly cycles, there can be multiple 
consecutive semi-diurnal cycles that do not emerge from within the stress shadow of the previous fortnightly peak stress (Fig. 3c). Seismicity rates overall are 10\% higher during the phases of the fortnightly cycle in which the APS is most frequently exceeded (Fig. $3 d, 3 e)$. However, the correlation between seismicity and peak semi-diurnal stress is only evident during the waning fortnightly cycle, when APS is exceeded less often, and overall seismicity rates are lower (Fig. 3b, 3d). The effect of more frequently exceeding APS appears to be the dominant driver of the overall seismicity rate, which is secondarily driven by the semi-diurnal tides (Fig. 3d).

The sensitivity of seismicity to APS can be considered in terms of 'memory' of previous stress states. Many different materials, including Earth materials, exhibit a memory of past stress conditions. The Kaiser Effect (Kaiser, 1959; Kurita and Fujii, 1979 ) is a stress threshold effect where intact rock is fractured only when APS has been exceeded, which is manifest in elevated levels of acoustic emissions (AE), or earthquakes. Using the rate and state model of Beeler and Lockner (2003), shear resistance is the combined effect of shear resistance to slip and shear resistance necessary to break unfractured rock. The ratio of fractured to unfractured crosssectional area is modeled as proportional to total slip. However, if the breaking of unfractured rock only or preferentially occurs when APS is exceeded, then either slip is suppressed when stress is below APS or the assumption of a linear relationship between total slip and area of fractured material is incorrect. When loading is uniform, the system is always exceeding APS rather than only during short time intervals so intact rock is always being fractured. According to the rate and state equations, during a period of rapid fracturing of rock, the associated fault weakening reduces the time-to- 
316 failure and the sensitivity of time-to-failure on stress variations. If there is a Kaiser

317 Effect, this could explain both the increase in seismicity and undetected sensitivity to semi-diurnal stress variations during rising fortnightly tides. Once nucleation is initiated on a fault, it is in a state of elevated stress-sensitive creep. Within a stress shadow, the only sites that fail are those already experiencing stress-driven creep even if weakening is muted. Peak seismicity at these sites should correlate with local peak stress even when the stress is below APS (Bartlow et al., 2012; Beeler and Lockner, 2003; Li and Nordlund, 1993; Li et al., 2010; Liu et al., 2010). This would explain why earthquakes are observed during all parts of the tidal cycles, but preferentially at higher stresses within stress shadows.

An important application of tidal triggering of earthquakes is as an indicator of pore pressure and frictional behavior (Fig. 3). LFEs near Parkfield (but 10-15 km deeper than the earthquakes studied here) are triggered during peak tidal shear stresses (Thomas et al., 2009), but show little sensitivity to much larger increases in extensional stress. This suggests that the ratio of tidal shear stress to effective normal stress is high due to low effective normal stress resulting from near lithostatic pore pressure (see equation 1 in Thomas et al.(2012) and Dieterich (2007)),

$$
R(\phi)=r * \exp \left(\frac{C \cos \phi}{a \sigma}\right)
$$

where $\phi$ is phase angle, $R$ is seismicity rate, $r$ is average seismicity rate, $C$ is periodic Coulomb stress amplitude, $a$ is the rate constitutive parameter (0.02) (Dieterich, 2007; Thomas et al., 2012), and $\sigma$ is effective normal stress. The seismicity rate is sensitive to the effective normal stress and not to the ratio of shear to normal stress. An explanation for why LFEs show little sensitivity to much larger reductions in normal 
stress is that conditions are anisotropic and Skempton's coefficient is very close to one on the deep SAF (eq. 1), which is common in low effective normal stress conditions

341 (Beeler et al., 2013; Green and Wang, 1986; Hawthorne and Rubin, 2010; Houston, 2015). Any increase in extensional stress would be countered with an equal reduction in pore pressure resulting in no net change in effective normal stress or Coulomb stress (Fig. 4). Alternatively, very low $\mu$ could produce the same result.

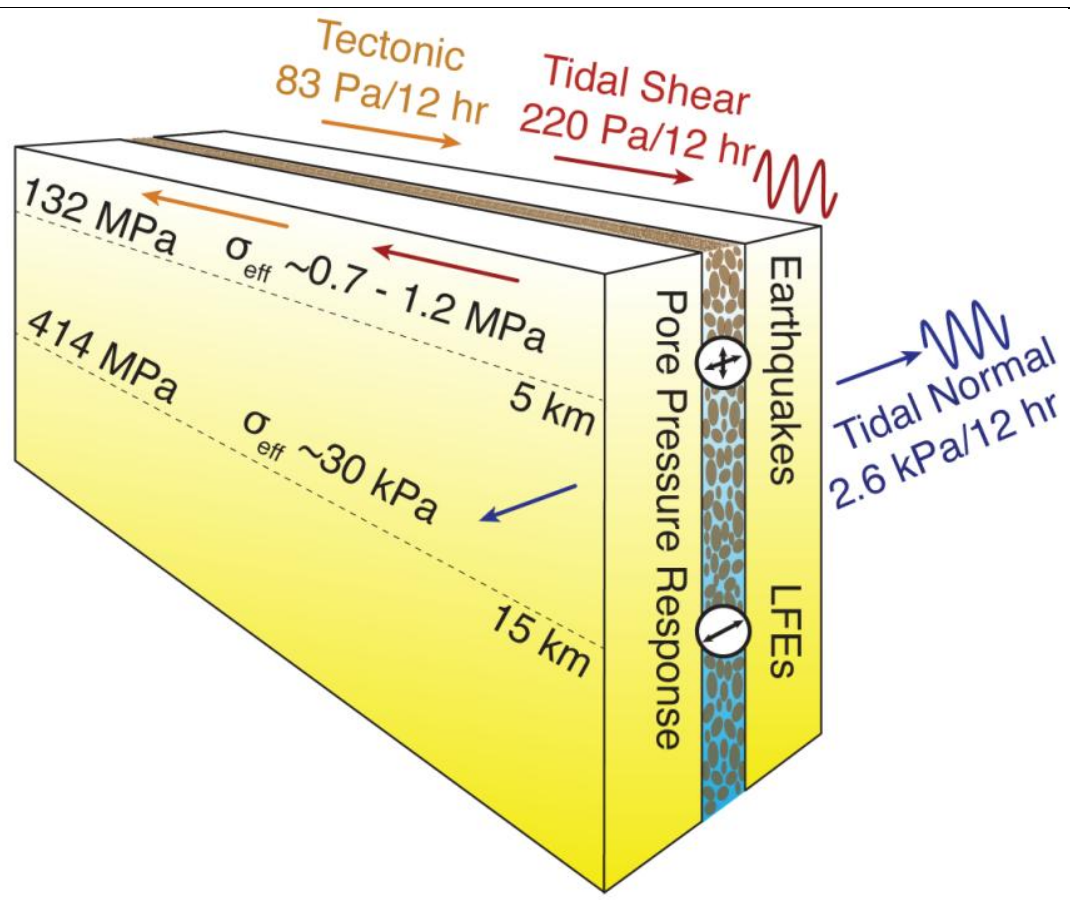

Fig. 4. Interpretation. The San Andreas Fault is loaded by tectonic shear stress (orange), tidal shear stress (red), and tidal normal stress (blue). Earthquakes in the upper crust are correlated with extensional tidal normal stress. Low frequency earthquakes in the middle crust are correlated with tidal shear stress. Lithostatic pressure, effective normal stress, and depth are indicated along dashed lines. In the middle crust, pore pressures respond to tidal normal stress with anisotropic poroelastic behavior, reducing the effective normal stress and cancelling the contribution to Coulomb stress. In the upper crust poroelastic behavior is at least partially 
isotropic and normal stress contribution to Coulomb stress is only partially canceled. In short, tidal triggering of earthquakes transition from occurring during peak tidal extensional normal stresses to peak tidal shear stresses as pore pressures increase from below lithostatic pressure to near lithostatic pressure.

We can estimate effective normal stress in the same way as Thomas et al., (2012) though in our case the result depends on Skempton's coefficient, B due to the interaction between tidal normal stresses and pore pressure. In the anisotropic case, B $<0.6$ due to the constraint of equation (5), but this also requires $\mu$ to be higher than it is measured in the creeping section of the SAF to the north of Parkfield (Carpenter et al., 2015). B is rarely measured to be below 0.4 in Earth materials, e.g. Rice and Cleary (1976), leaving a small window where there are somewhat reasonable values for both $\mu$ and $B$. Using $B=0.5$ and $\mu=0.8$ with equation (1), the effective normal stress is 700 $354 \mathrm{kPa}$, an order of magnitude higher than the $37 \mathrm{kPa}$ determined for the middle crust in the vicinity of LFEs and tremor (Thomas et al., 2012). In the isotropic case B is not well constrained but has only a small effect on the results for effective normal stress which range from 1.2 $\mathrm{MPa}$ for $\mathrm{B}=0.5$ to $1.0 \mathrm{MPa}$ for $\mathrm{B}=1$; both values are two orders of magnitude higher than that for the middle crust. As the stress drop for $M_{w} 6$ earthquakes on the SAF are at least $1 \mathrm{MPa}$, our preferred model is the isotropic one. Pore pressure and effective normal stress are important parameters in frictional systems such as those in active tectonic regions as well as engineered environments associated with hydraulic fracturing, wastewater injection, and $\mathrm{CO} 2$ sequestration. Based on results presented here, we posit that tidal triggering of earthquakes should transition from occurring during peak tidal extensional normal stresses to peak tidal shear stresses as pore pressures increase from below lithostatic pressure to near 
366 lithostatic pressure. If this were the case it could be highly useful in inferring time or

367 space-dependent pore pressure and poroelastic behavior in both reservoir and tectonic

368 environments.

369 The detection of tidal triggering is made possible by the interstation seismic

370 coherence method, which focuses on earthquake detection at the expense of precise

371 locations. This method offers a new and sensitive means to probe earthquake clustering

372 behavior and the time-varying stress state of both tectonic and injection-activated faults. 


\section{References}

Agnew, D.C., 1997. NLOADF: A program for computing ocean-tide loading. J Geophys Res 102, 5109-5110, doi: 10.1029/96jb03458.

Bartlow, N.M., et al., 2012. Laboratory triggering of stick-slip events by oscillatory loading in the presence of pore fluid with implications for physics of tectonic tremor. J Geophys Res 117, doi: 10.1029/2012jb009452.

Beeler, N.M., Lockner, D.A., 2003. Why earthquakes correlate weakly with the solid Earth tides: Effects of periodic stress on the rate and probability of earthquake occurrence. J Geophys Res 108, doi: 10.1029/2001jb001518.

Beeler, N.M., et al., 2000. Pore fluid pressure, apparent friction, and Coulomb failure. J Geophys Res 105, 25533-25542, doi: 10.1029/2000jb900119.

Beeler, N.M., et al., 2013. Inferring fault rheology from low- frequency earthquakes on the San Andreas. J Geophys Res 118, 5976-5990, doi: 10.1002/2013jb010118.

Brinkman, B.A.W., et al., 2015. Probing failure susceptibilities of earthquake faults using small-quake tidal correlations. Nat Commun 6, doi: 10.1038/ncomms7157.

Brodsky, E.E., van der Elst, N.J., 2014. The Uses of Dynamic Earthquake Triggering. Annu Rev Earth PI Sc 42, 317-339, doi: 10.1146/Annurev-Earth-060313-054648.

Carpenter, B.M., et al., 2015. Frictional properties of the active San Andreas Fault at SAFOD: Implications for fault strength and slip behavior. J Geophys Res 120, 5273-5289, doi: 10.1002/2015jb011963.

Cochran, E.S., et al., 2004. Earth tides can trigger shallow thrust fault earthquakes. Science 306, 1164-1166, doi: 10.1126/science.1103961. 
397 Delorey, A.A., et al., 2015. Cascading elastic perturbation in Japan due to the $2012 \mathrm{Mw}$

8.6 Indian Ocean Earthquake. Science Advances 1, e1500468, doi: 10.1126/sciadv.1500468.

Dieterich, J.H., 1987. Nucleation and Triggering of Earthquake Slip - Effect of Periodic Stresses. Tectonophysics 144, 127-139, doi: 10.1016/0040-1951(87)90012-6.

Dieterich, J.H., 2007. Applications of rate- and state-dependent friction to models of fault slip and earthquake occurence. Elsevier, New York

Emter, D., 1997. Tidal triggering of earthquakes and volcanic events. Lect Notes Earth Sci 66, 293-309

Field, E.H., et al., 2014. Uniform California Earthquake Rupture Forecast, Version 3 (UCERF3)-The Time-Independent Model. B Seismol Soc Am 104, 1122-1180

Gomberg, J., et al., 2004. Earthquake nucleation by transient deformations caused by the $M=7.9$ Denali, Alaska, earthquake. Nature 427, 621-624, doi: 10.1038/Nature02335.

Gonzalez-Huizar, H., et al., 2012. Remote triggered seismicity caused by the 2011, M9.0 Tohoku-Oki, Japan earthquake. Geophys Res Lett 39, L10302, doi: 10.1029/2012GL051015.

Green, D.H., Wang, H.F., 1986. Fluid Pressure Response to Undrained Compression in Saturated Sedimentary-Rock. Geophysics 51, 948-956, doi: 10.1190/1.1442152. Hawthorne, J.C., Rubin, A.M., 2010. Tidal modulation of slow slip in Cascadia. J Geophys Res 115, doi: 10.1029/2010jb007502. 
418 Hill, D.P., et al., 1993. Seismicity Remotely Triggered by the Magnitude 7.3 Landers, California, Earthquake. Science 260, 1617-1623, doi: 10.1126/Science.260.5114.1617.

421 Houston, H., 2015. Low friction and fault weakening revealed by rising sensitivity of tremor to tidal stress. Nature Geoscience 8, 409-415, doi: 10.1038/Ngeo2419.

Johnson, P.A., et al., 2013. Acoustic emission and microslip precursors to stick-slip failure in sheared granular material. Geophys Res Lett 40, 5627-5631, doi: 10.1002/2013gl057848.

Kaiser, E.J., 1959. A study of acoustic phenomena in tensile test. Technische Hochschule Munchen

Kim, A., Dreger, D.S., 2008. Rupture process of the 2004 Parkfield earthquake from near-fault seismic waveform and geodetic records. J Geophys Res 113, doi: 10.1029/2007jb005115.

Kurita, K., Fujii, N., 1979. Stress Memory of Crystalline Rocks in Acoustic-Emission. Geophys Res Lett 6, 9-12, doi: 10.1029/GL006i001p00009.

Li, C., Nordlund, E., 1993. Experimental-Verification of the Kaiser Effect in Rocks. Rock Mech Rock Eng 26, 333-351, doi: 10.1007/Bf01027116.

Li, Y.H., et al., 2010. Experimental and theoretical analysis on the procedure for estimating geo-stresses by the Kaiser effect. Int J Min Met Mater 17, 514-518, doi: 10.1007/s12613-010-0351-3.

Liu, J.P., et al., 2010. Experimental study on load/unload response ratio and Kaiser effect when rocks under cycling load. Rock Stress and Earthquakes, 205-209 
Mcnutt, S.R., Beavan, R.J., 1981. Volcanic Earthquakes at Pavlof Volcano Correlated with the Solid Earth Tide. Nature 294, 615-618, doi: 10.1038/294615a0.

Metivier, L., et al., 2009. Evidence of earthquake triggering by the solid earth tides. Earth Planet Sc Lett 278, 370-375, doi: 10.1016/J.Epsl.2008.12.024.

Murray, J., Langbein, J., 2006. Slip on the San Andreas fault at Parkfield, California, over two earthquake cycles, and the implications for seismic hazard. B Seismol Soc Am 96, S283-S303, doi: 10.1785/0120050820.

Obara, K., 2002. Nonvolcanic deep tremor associated with subduction in southwest Japan. Science 296, 1679-1681, doi: 10.1126/Science.1070378.

Rice, J.R., Cleary, M.P., 1976. Some Basic Stress Diffusion Solutions for FluidSaturated Elastic Porous-Media with Compressible Constituents. Rev Geophys 14, 227-241, doi: 10.1029/RG014i002p00227.

Rydelek, P.A., et al., 1988. Tidal Triggering of Earthquake Swarms at Kilauea Volcano, Hawaii. J Geophys Res 93, 4401-4411, doi: 10.1029/JB093iB05p04401.

Shelly, D.R., Hardebeck, J.L., 2010. Precise tremor source locations and amplitude variations along the lower-crustal central San Andreas Fault. Geophys Res Lett 37, doi: 10.1029/2010gl043672.

Stroup, D.F., et al., 2007. Pulse of the seafloor: Tidal triggering of microearthquakes at 9 degrees 50'N East Pacific Rise. Geophys Res Lett 34, doi: $10.1029 / 2007 g \mid 030088$.

Tanaka, S., 2010. Tidal triggering of earthquakes precursory to the recent Sumatra megathrust earthquakes of 26 December 2004 (M-w 9.0), 28 March 2005 (M-w 
8.6), and 12 September 2007 (M-w 8.5). Geophys Res Lett 37, L02301, doi: $10.1029 / 2009 g \mid 041581$.

464 Tanaka, S., 2012. Tidal triggering of earthquakes prior to the 2011 Tohoku-Oki earthquake (M-w 9.1). Geophys Res Lett 39, doi: 10.1029/2012gl051179.

466

467

468

469

470

471

472

473

474

475

476

477

478

479

480

481

482

Tanaka, S., et al., 2002a. Evidence for tidal triggering of earthquakes as revealed from statistical analysis of global data. J Geophys Res 107, doi: 10.1029/2001jb001577.

Tanaka, S., et al., 2002b. Spatio-temporal variation of the tidal triggering effect on earthquake occurrence associated with the 1982 South Tonga earthquake of Mw 7.5. Geophys Res Lett 29, doi: 10.1029/2002gl015386.

Thomas, A.M., et al., 2012. Tidal triggering of low frequency earthquakes near Parkfield, California: Implications for fault mechanics within the brittle-ductile transition. J Geophys Res 117, doi: 10.1029/2011jb009036.

Thomas, A.M., et al., 2009. Tremor-tide correlations and near-lithostatic pore pressure on the deep San Andreas fault. Nature 462, 1048-1051, doi: 10.1038/Nature08654.

Tolstoy, M., et al., 2002. Breathing of the seafloor: Tidal correlations of seismicity at Axial volcano. Geology 30, 503-506, doi: 10.1130/00917613(2002)030<0503:Botstc>2.0.Co;2.

Townend, J., Zoback, M.D., 2000. How faulting keeps the crust strong. Geology 28, 399-402, doi: 10.1130/0091-7613(2000)28<399:Hfktcs>2.0.Co;2. 
01

Tsuruoka, H., et al., 1995. Statistical Test of the Tidal Triggering of Earthquakes Contribution of the Ocean Tide Loading Effect. Geophys J Int 122, 183-194, doi: 10.1111/j.1365-246X.1995.tb03546.x.

van der Elst, N.J., et al., 2016. Fortnightly modulation of San Andreas tremor and lowfrequency earthquakes. Procedings of the National Academy of Science 113, 8601-8605, doi: 10.1073/pnas.1524316113.

van der Elst, N.J., et al., 2013. Enhanced Remote Earthquake Triggering at FluidInjection Sites in the Midwestern United States. Science 341, 164-167, doi: 10.1126/Science.1238948.

Vidale, J.E., et al., 1998. Absence of earthquake correlation with Earth tides: An indication of high preseismic fault stress rate. J Geophys Res 103, 24567-24572, doi: 10.1029/98jb00594.

Wech, A.G., Creager, K.C., 2008. Automated detection and location of Cascadia tremor. Geophys Res Lett 35, doi: 10.1029/2008gl035458.

Wilcock, W.S.D., 2001. Tidal triggering of micro earthquakes on the Juan de Fuca Ridge. Geophys Res Lett 28, 3999-4002, doi: 10.1029/2001gl013370.

Wilcock, W.S.D., 2009. Tidal triggering of earthquakes in the Northeast Pacific Ocean. Geophys J Int 179, 1055-1070, doi: 10.1111/J.1365-246x.2009.04319.X. 


\section{Acknowledgments}

504 Waveform data, metadata, or data products for this study were accessed through the

505 Northern California Earthquake Data Center (NCEDC), doi:10.7932/NCEDC and

506 Southern California Earthquake Data Center (SCEDC) doi:10.7909/C3WD3xH1. The

507 LFE catalog is provided by David Shelly and is an update to the Shelly and Hardebeck

508 (Shelly and Hardebeck, 2010) catalog. The authors wish to thank Earl Lawrence for

509 consultations on statistical methods. AD and PJ received support from the U.S.

510 Department of Energy (DOE) through the subTER Crosscut and institutional support

511 from Los Alamos National Laboratory. NV received support from the Mendenhall

512 postdoctoral program for this study.

513 Author Contributions:

514 AD developed the earthquake detection method, performed the statistical analysis on

515 the earthquake catalog, and contributed to the interpretation of the results. NVDE

516 contributed to the statistical analysis of the earthquake catalog and the interpretation of

517 the results. PAJ guided the direction of this study and contributed to the presentation

518 and interpretation of the results. All authors participated in the writing of the manuscript.

519 Competing Financial Interests statement:

520 The authors have no competing interests. 